\title{
Efeito do número de demonstrações na aquisição de uma habilidade motora: um estudo exploratório
}

\author{
Alessandro Bruzi \\ Leandro Palhares \\ João Fialho \\ Rodolfo Benda \\ Herbert Ugrinowitsch
}

https://doi.org/10.5628/rpcd.06.02.179

\author{
Universidade Federal de Minas Gerais \\ Escola de Educação Física, Fisioterapia e Terapia Ocupacional \\ Grupo de Estudos em Desenvolvimento e Aprendizagem \\ Motora \\ Belo Horizonte - MG \\ Brasil
}

\section{RESUMO}

O efeito de diferentes números de demonstrações na aquisição de habilidades motoras ainda não está claro. Isto incentivou a investigação do efeito do número de demonstrações na aprendizagem do arremesso de dardo de salão. Quatorze sujeitos foram distribuídos aleatoriamente em 4 grupos: uma demonstração (D1), duas (D2), quatro (D4) e oito (D8). O experimento constou de três fases: aquisição, com 70 tentativas do arremesso "profissional", a 2,37m do alvo; teste de transferência após 3 minutos, com 10 tentativas da pegada em "V", a 3,37m; e teste de retenção da aquisição, com 10 tentativas, após 10 minutos. Na análise do padrão de movimento, pela moda, não se detectou diferença significativa intergrupos $(p>0,05)$ e detectou-se, no índice de variabilidade do padrão de movimento (IVP), diferença intergrupos no bloco 1 do teste de retenção, sendo 4 e 8 demonstrações superiores. Os resultados mostram que todos os grupos foram semelhantes na aprendizagem da tarefa. Além disso, 4 e 8 demonstrações apresentaram uma maior flexibilidade no padrão de movimento.

Palavras-chave: aprendizagem motora, habilidade motora, número de demonstrações.

\begin{abstract}
Effect of the number of demonstrations on a motor skill learning: An exploratory study

The effect of the number of demonstrations on motor skill learning isn't clear. This originated the present research about the effect of the number of demonstrations on a dart throwing learning task. Fourteen subjects were randomly divided into four groups: one (D1), two (D2), four (D4) and eight (D8) demonstrations. This study had three phases: (1) acquisition, with 70 trials of "professional" dart throwing, $2,37 \mathrm{~m}$ far from the target; (2) transfer test after three minutes, 10 trials with " $V$ " dart throwing, 3,37m far from the target; (3) acquisition retention test, 10 trials, after ten minutes. Kruskal-Wallis test didn't show significant differences $(p>0,05)$ among the movement patterns of the different groups. However, differences among the movement pattern variability index (IVP) of the different groups in the first block of retention test were found, corresponding the best performances to $D 4$ and $D 8$ groups. Results also evidentiated that all groups were similar on the motor skill learning. Despite of this, groups D4 and D8 showed more movement pattern flexibility.
\end{abstract}

Key Words: number of demonstrations, motor skill, motor learning. 


\section{INTRODUÇÃO}

A prática de modalidades esportivas, visando os diversos fins, objetiva o alcance de níveis máximos ou ótimos de desempenho (21). Independente do contexto, o desempenho desejado é alcançado por meio de ações habilidosas. Desta maneira, é essencial investigar a aprendizagem de habilidades esportivas, processo que envolve prática e informação, tais como feedback, instrução verbal e demonstração (16). Dessa maneira, é possível identificar a importância da demonstração como variável influente na aquisição de habilidades motoras, quando comparada à instrução verbal $(1,19)$. A demonstração transmite ao observador características espaciais e temporais do movimento que o ajudam a desenvolver uma representação cognitiva da ação, que é usada na produção do movimento e serve como padrão para detecção e correção de erros $(5,6,7,8,17,18)$. Existem algumas variáveis envolvidas com o estudo de demonstração, sendo algumas delas o nível de desempenho do modelo, o nível de desenvolvimento motor do aprendiz ou ainda o número de demonstrações fornecido. O número de demonstrações é entendido como um aspecto importante, pois pode oportunizar um maior número de chances ao aprendiz de identificar aspectos cruciais da habilidade motora (15). Contudo, poucos estudos que avaliaram o efeito de diferentes números de demonstrações analisaram a eficiência desta variável na qualidade do padrão de movimento $(8,10,14)$. Outro fato comum nestes estudos é a utilização de tarefas de laboratório como, por exemplo, a tarefa de subir a escada de Bachman (10), de timing coincidente (26) e da simulação da ação remar $(8,14)$. Em geral, os resultados da maioria dos estudos mostraram que um maior número de demonstrações tem sido mais eficiente na aquisição de um padrão de movimento, quando comparado com um menor número de demonstrações.

Um problema desses estudos, realizados com tarefas de laboratório onde se enfatiza a fidedignidade dos resultados $(20,24)$, é que os seus resultados não podem ser diretamente aplicados em situações reais de ensino-aprendizagem. Dessa forma, faz-se necessário testar os resultados obtidos nas pesquisas de laboratório em situações que se aproximem mais das situações de ensino-aprendizagem $(9,11,20,23)$.
Sendo assim, o objetivo deste estudo foi o de investigar o efeito de diferentes números de demonstrações na aprendizagem do arremesso de dardo de salão.

\section{MATERIAL E MÉTODOS Amostra}

Participaram do estudo 14 universitários voluntários com consentimento livre e esclarecido, de ambos os gêneros, na faixa etária entre 18 e 35 anos e sem experiência prévia na tarefa.

\section{Tarefa e instrumentos}

A tarefa utilizada foi o arremesso de dardo de salão. Foram utilizadas duas variações do arremesso de dardo com a mão dominante: I) arremesso de dardo com pegada "profissional", no qual o sujeito se posicionou de lado em relação ao alvo e o dardo era apoiado nas faces anteriores dos dedos polegar, indicador, médio e mínimo e na face posterior do dedo anelar, e II) arremesso de dardo com pegada em "V", no qual o sujeito se posicionou de frente para o alvo e o dardo era apoiado entre os dedos indicador e médio, totalmente flexionados, e na face anterior do dedo polegar.

Foi utilizado um instrumento para medida de desempenho que continha duas estruturas: a) um equipamento profissional para arremesso de dardo de salão contendo um alvo de sisal e metal, com 12 círculos circunscritos nas cores vermelha (centro), preta e branca para pontuação que variava de 1 (extremidade) a 12 pontos (centro), fixo a um tripé e seis dardos com ponta metálica, e b) duas filmadoras MC Panasonic, modelo AG 196, UP/VHS, para filmagem das tentativas da fase de aquisição e do teste de retenção, posicionadas no plano sagital e a $45^{\circ}$ em relação ao executante.

\section{Procedimentos e delineamento experimental}

A coleta de dados foi realizada em um ginásio de atividades da Escola de Educação Física, Fisioterapia e Terapia Ocupacional da Universidade Federal de Minas Gerais, no qual os equipamentos de coleta foram dispostos. Os sujeitos se encaminhavam ao local de coleta, eram informados quanto à sua dinâmica e, após terem assinado o consentimento livre e esclarecido, eram distribuídos aleatoriamente em um dos grupos experimentais: D1 (uma demonstração), 
D2 (duas demonstrações), D4 (quatro demonstrações) ou D8 (oito demonstrações). Após as explicações e as demonstrações tinha início a fase de aquisição com um comando sonoro "filma", que servia para que os sujeitos adotassem a posição inicial do arremesso com pegada "profissional" e para que ambas filmadoras fossem acionadas. Após 3 segundos de filmagem era dado o comando de "prepara" para que o sujeito realizasse o arremesso. Ao final do arremesso, a filmagem era interrompida e era fornecido ao sujeito o conhecimento do resultado (CR) sobre o escore obtido na tentativa. Após o fornecimento do CR era disponibilizado outro dardo para a tentativa seguinte, dando seqüência ao procedimento durante toda a fase de aquisição (70 execuções). Ao término da fase de aquisição houve um intervalo de três minutos para ajuste do equipamento e para o fornecimento da instrução verbal sobre a nova tarefa do teste de transferência (TT) com 10 execuções: arremesso de dardo com pegada em "V". Em seguida teve início o teste de transferência sem o fornecimento de CR e sem utilização de filmagem, tendo apenas o comando de "prepara" para início da tentativa. Ao final do teste de transferência houve dez minutos de intervalo para realização do teste de retenção (TR) da aquisição, com a utilização do arremesso com pegada "profissional", sendo realizadas mais 10 execuções com as filmagens.
Quadro 1. Grupos e fases do experimento.

\begin{tabular}{|c|c|c|c|}
\hline $\begin{array}{l}\text { Fases } \\
\text { Grupo }\end{array}$ & $\begin{array}{c}\text { Fase de } \\
\text { Aquisição }\end{array}$ & $\begin{array}{c}\text { Teste de } \\
\text { Transferência }\end{array}$ & $\begin{array}{c}\text { Teste de } \\
\text { Retenção }\end{array}$ \\
\hline $\begin{array}{l}\text { D1 luma } \\
\text { demonstração }\end{array}$ & 70 tentativas & 10 tentativas & 10 tentativas \\
\hline $\begin{array}{l}\mathrm{D} 2 \text { (duas } \\
\text { demonstrações }\end{array}$ & $\begin{array}{c}\text { pegada } \\
\text { "profissional" }\end{array}$ & $\begin{array}{l}\text { pegada } \\
\text { em "V" }\end{array}$ & $\begin{array}{c}\text { pegada } \\
\text { "profissional" }\end{array}$ \\
\hline $\begin{array}{l}\text { D4 (quatro } \\
\text { demonstrações }\end{array}$ & & 3 minutos & 10 minutos \\
\hline $\begin{array}{l}\text { D8 loito } \\
\text { demonstrações }\end{array}$ & & $\begin{array}{c}\text { após a } \\
\text { aquisição }\end{array}$ & $\begin{array}{c}\text { após a } \\
\text { retenção }\end{array}$ \\
\hline
\end{tabular}

\section{Decodificação dos dados para análise do padrão de movimento}

As filmagens de 80 tentativas por sujeito (70 da fase de aquisição e 10 do teste de retenção) foram analisadas de acordo com o instrumento para avaliação do padrão de movimento (Quadro 2). Para isso, foi utilizado uma TV e um vídeo cassete, no qual as imagens foram reproduzidas em velocidade normal e em câmera lenta. Antes da decodificação dos dados o instrumento foi testado em um estudo piloto, com três observadores, e os resultados mostraram um índice de concordância intra-observadores de 0.87 e inter-observadores de 0.94 (22). Concluído este procedimento, a decodificação dos dados foi realizada por apenas uma pessoa.

\begin{tabular}{|c|c|c|c|}
\hline \multicolumn{4}{|c|}{ CRITÉRIO PARA ANÁUSE DO PADRĀO DE MOVIMENTO - ARREMESSO DE DARDO DE SALÃO "PROFISSIONAL" } \\
\hline \multirow[b]{2}{*}{ ASPECTOS } & \multicolumn{3}{|c|}{\begin{tabular}{|c|} 
PONTUACÃO \\
\end{tabular}} \\
\hline & Pontuação 3 & Pontuação 2 & Pontuação 1 \\
\hline $\begin{array}{l}\text { Aspecto } 1 \\
\text { (membros } \\
\text { inferiores) }\end{array}$ & $\begin{array}{l}\text { Membros inferiores paralelos } \\
\text { entre si eà linha limitrofe de } \\
\text { arremesso. }\end{array}$ & $\begin{array}{l}\text { Sem um dos pés em entre } \\
\text { si elou com um dos pés } \\
\text { parcialmente fora do solo. }\end{array}$ & $\begin{array}{l}\text { Pés náo paralelos entr si, } \\
\text { pés parcialmente fora do } \\
\text { solo, um dos pés fora do } \\
\text { solo, pés fora do solo, ou } \\
\text { a combinaçáo entre estas } \\
\text { situraçōes. }\end{array}$ \\
\hline $\begin{array}{l}\text { Aspecto } 2 \\
\text { (tronco) }\end{array}$ & Tronco perpendicular ao alvo. & Giro parcial no tronco. & $\begin{array}{l}\text { Tronco de frente para } \\
\text { o alvo. }\end{array}$ \\
\hline $\begin{array}{l}\text { Aspecto } 3 \\
\text { (ombro, } \\
\text { braço, } \\
\text { cotovelo, } \\
\text { antebraço) }\end{array}$ & $\begin{array}{l}\text { Ombro em abdução como } \\
\text { braço paralelo ao solo e } \\
\text { cotovelo flexionado, devendo } \\
\text { o cotovelo se estender } \\
\text { totalmente no final do } \\
\text { arremesso. }\end{array}$ & $\begin{array}{l}\text { Ombros abduzidos ou } \\
\text { aduzidos desde que o braço } \\
\text { não esteja perpendicular ao } \\
\text { solo. }\end{array}$ & $\begin{array}{l}\text { Ombros abduzidos ou } \\
\text { aduzidos com braço } \\
\text { perpendicular ao solo. }\end{array}$ \\
\hline $\begin{array}{r}\text { Aspecto } 4 \\
\text { (pegada) }\end{array}$ & $\begin{array}{l}\text { Pegada "profissionar: dardo } \\
\text { apoiado na face anterior pelos } \\
\text { dedos indicador, médio, e } \\
\text { polegar e na face posterior } \\
\text { pelo dedo anelar. A posiçăo } \\
\text { deve ser mantida durante } \\
\text { o arremesso. } \\
\end{array}$ & $\begin{array}{l}\text { Não utilizar um dos dedos } \\
\text { como apoio para o dardo ou } \\
\text { utilizar outro dedo, que não o } \\
\text { anelar, para apoiar o dardo } \\
\text { na face arterior. }\end{array}$ & $\begin{array}{l}\text { Utilizar dois ou mais dedos } \\
\text { de forma inadequada à } \\
\text { pegada "profissional". }\end{array}$ \\
\hline
\end{tabular}

Quadro 2. Instrumento para avaliação do padrão de movimento do arremesso de dardo com pegada "profissional" (4). 


\section{Tratamento estatístico}

Para cada sujeito foram analisadas duas varáveis dependentes: uma quantitativa, que avaliou o desempenho (escore) obtido pela pontuação dos arremessos no alvo, e outra, qualitativa, que avaliou o processo de execução (padrão de movimento), decodificado a partir do instrumento proposto por Bruzi et al. (4). Para análise do escore foi calculada a média e o desvio-padrão em blocos de 5 tentativas. Para análise do padrão de movimento, foi utilizada a moda de cada aspecto (Quadro 2) em blocos de 5 tentativas. Foram utilizadas comparações inter e intragrupo da moda de cada aspecto, e também da soma da moda de cada aspecto, obtendo um valor global do padrão de movimento. Para análise da variabilidade do padrão de movimento foi utilizado o Índice de Variabilidade do Padrão (IVP) por aspecto, proposto por Barela \& Barela (3), já utilizado por Gimenez et al. (13):

$$
\text { IVP }=\frac{\text { Número de Padrões Diferentes }}{\text { Número Total de Tentativas }}
$$

No presente estudo, o índice mais baixo corresponde a zero e o índice mais alto a 0.6. Para esta análise foram utilizados blocos de 5 tentativas para as comparações inter e intragrupo dos IVPs de cada aspecto, e também inter e intragrupo da média entre os IVPs de cada aspecto, ou seja, o IVP que representou a habilidade.

Para analisar a existência de diferença intergrupos foi utilizado, tanto para o escore quanto para análise do padrão de movimento, o teste não-paramétrico de Kruskal-Wallis. Para análise de diferença intragrupos foi utilizado o teste não-paramétrico de Friedman. Os dados foram analisados pelo pacote Statistica for Windows 6.0 e manteve-se um nível de significância de $\mathrm{p} \leq 0,05$. Nos casos que existiram diferenças foi utilizado o procedimento de Bonferroni para identificar onde ocorreram as diferenças intergrupos, nos testes de transferência e retenção, e intragrupos, do último bloco da aquisição para os testes (25). Quando não foram identificadas as diferenças devido ao ajuste do "p" referente ao número de medidas repetidas, o procedimento utilizado foi o de observação das curvas dos gráficos.

\section{RESULTADOS}

Ao realizar a análise descritiva dos resultados, é possível identificar a efetiva potência da variável demonstração no padrão de movimento e que diferentes números de demonstrações promoveram diferença intra e intergrupos quanto à variabilidade do padrão formado.

\section{Fase de aquisição}

$\mathrm{Na}$ análise inferencial intragrupos da média do escore, o teste de Friedman identificou diferença significativa para o D1 $[\mathrm{F}(\mathrm{n}=4, \mathrm{df}=13)=22,903$, $\mathrm{p}<0,04]$. Ao observar a Figura 1 é possível supor que, provavelmente, o D1 aumentou sua precisão do bloco 1 para os blocos 7, 8, 10 e 13. Os demais grupos se mantiveram semelhantes com relação à média do escore $(p>0,05)$.

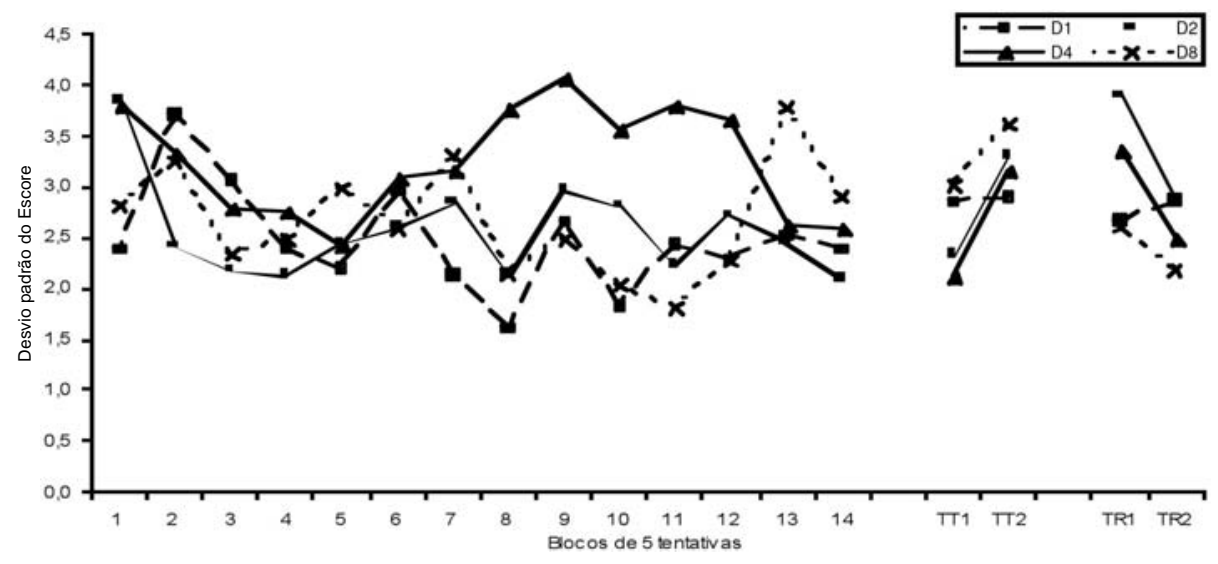

Figura 1. Média do escore em blocos de 5 tentativas.
Na análise do desviopadrão do escore, o teste de Friedman não identificou alterações na variabilidade $(p>0,05)$ em nenhum dos grupos experimentais durante essa fase do experimento (Figura 2). 


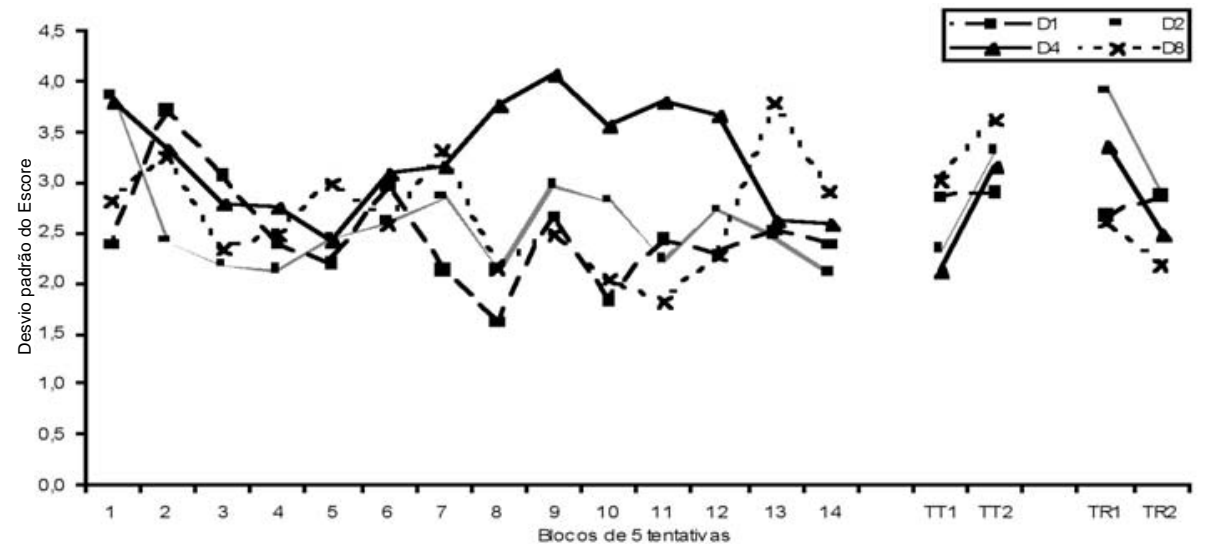

Com relação ao padrão de movimento, na análise isolada da moda de cada aspecto, o teste de Friedman não identificou diferença intragrupos $(p>0,05)$, o mesmo ocorrendo na somatória das modas de cada aspecto (Quadro 3). Com relação à variabilidade do padrão de movimento, na análise da média entre os IVPs de cada aspecto, o teste de Friedman identificou diferenças para o D1 $[\mathrm{F}(\mathrm{n}=4, \mathrm{df}=14)=46,899$, $\mathrm{p}<0,01]$, para o D2 $[\mathrm{F}(\mathrm{n}=3, \mathrm{df}=14)=26,637$, $\mathrm{p}<0,02]$ e para o D8 $[\mathrm{F}(\mathrm{n}=4, \mathrm{df}=14)=38,396$, $\mathrm{p}<0,01]$. Ao observar o Quadro 4, é possível supor que, provavelmente, o D1 reduziu sua variabilidade sendo o bloco 1 superior aos blocos 2, 4, 11 e 12, o D2 reduziu sua variabilidade do bloco 1 para os blo$\cos 7,11$ e 12 e, no D8, os blocos 3, 5, 8, 9, 10, 11, 13 e 14 tiveram variabilidade inferior aos demais blocos (Quadro 4). O teste utilizado não identificou alteração nessa medida no grupo D4 $(p>0,05)$.

Quadro 3. Avaliação do padrão de movimento (PM] pela moda do somatório das modas dos aspectos e pelas modas de cada aspecto.

\begin{tabular}{|c|c|c|c|c|c|c|c|c|c|c|c|c|c|c|c|c|c|}
\hline \multirow{2}{*}{\multicolumn{2}{|c|}{ AVALIAÇĀO DO PM }} & \multicolumn{16}{|c|}{ BLOCOS DE TENTATIVAS } \\
\hline & & \multirow{2}{*}{$\begin{array}{l}1 \\
9\end{array}$} & \multirow{2}{*}{$\frac{2}{6}$} & \multirow{2}{*}{$\frac{3}{7}$} & \multirow{2}{*}{$\frac{4}{\star \star \star \star}$} & \multirow{2}{*}{$\frac{5}{\star \star \star}$} & \multirow{2}{*}{$\frac{6}{10}$} & \multirow{2}{*}{7} & \multirow{2}{*}{$\begin{array}{l}8 \\
9\end{array}$} & \multirow{2}{*}{$\frac{9}{\star \star \star}$} & \multirow{2}{*}{$\frac{10}{9}$} & \multirow{2}{*}{$\frac{11}{10}$} & \multirow{2}{*}{$\frac{12}{10}$} & \multirow{2}{*}{$\frac{13}{\star \star \star}$} & \multirow{2}{*}{$\frac{14}{9}$} & \multirow{2}{*}{$\frac{\text { TR1 }}{\star \star \star \star}$} & \multirow{2}{*}{$\frac{\text { TR2 }}{\star \star \star}$} \\
\hline Moda do & D1 & & & & & & & & & & & & & & & & \\
\hline \multirow{3}{*}{$\begin{array}{l}\text { Som atório das } \\
\text { Modas dos Aspectos }\end{array}$} & D2 & 9 & 10 & $\star \star \star \star$ & $\star \star \star \star$ & 8 & $\star \star \star \star$ & 8 & $\star \star \star \star$ & $\star \star \star$ & $\star \star \star \star$ & 9 & 9 & 9 & $\star \star \star \star$ & $\star \star \star$ & $\star \star \star \star$ \\
\hline & D4 & $\star \star \star \star$ & 8 & 8 & 8 & $\star \star \star \star$ & 8 & $\star \star \star \star$ & 8 & 8 & 8 & 7 & $\star \star \star *$ & $\star \star \star \star$ & $\star \star \star *$ & $\star \star \star \star$ & 8 \\
\hline & D8 & 10 & 8 & 9 & 8 & 8 & 9 & 9 & 9 & 9 & 9 & 9 & 9 & 9 & 9 & 9 & 9 \\
\hline \multirow[t]{4}{*}{ Moda do Aspecto 1} & D1 & 2 & 1 & 1 & 1 & 1 & 2 & 2 & 2 & 2 & 2 & 2 & 2 & 2 & 2 & 2 & 2 \\
\hline & D2 & 2 & 2 & 2 & 2 & 2 & 2 & 2 & 2 & 2 & 2 & 2 & 2 & 2 & 2 & 2 & 2 \\
\hline & D4 & 3 & 2 & 2 & 2 & 2 & 2 & 3 & 2 & 2 & 2 & 2 & 2 & 2 & 2 & 2 & 2 \\
\hline & D8 & 2 & 2 & 3 & 2 & 2 & 2 & 3 & 3 & 3 & 3 & 3 & 3 & 3 & 3 & 3 & 3 \\
\hline \multirow[t]{4}{*}{ Moda do Aspecto 2} & D1 & 3 & $\star \star \star \star$ & $\star \star \star \star$ & 3 & 3 & 3 & 3 & 3 & 3 & 3 & 3 & 3 & $\star \star \star \star$ & 3 & 3 & 3 \\
\hline & D2 & 3 & 3 & 3 & 3 & 3 & 3 & 3 & 3 & 3 & 3 & 3 & 3 & 3 & 3 & 3 & 3 \\
\hline & D4 & 3 & 3 & 3 & 3 & 3 & 3 & 3 & 3 & 3 & 3 & 2 & 3 & 3 & 3 & 3 & 3 \\
\hline & D8 & 2 & 2 & 2 & 2 & 2 & 2 & 2 & 2 & 2 & 2 & 2 & 2 & 2 & 2 & 2 & 2 \\
\hline \multirow[t]{4}{*}{ Moda do Aspecto 3} & D1 & 2 & 1 & 1 & 2 & 2 & 2 & 2 & 2 & 2 & 2 & 2 & 2 & 1 & 2 & 2 & 3 \\
\hline & D2 & 2 & 2 & 2 & 2 & 2 & 2 & 2 & 2 & 2 & 2 & 2 & 2 & 2 & $\star \star \star *$ & $\star \star \star$ & $\star \star \star \star$ \\
\hline & D4 & 2 & 2 & 2 & 2 & 2 & 2 & 2 & 2 & 2 & 2 & 2 & 2 & 2 & 2 & 2 & 2 \\
\hline & D8 & 1 & 1 & 1 & 1 & 1 & 2 & 1 & 1 & 1 & 1 & 1 & 1 & 1 & 1 & 1 & 1 \\
\hline \multirow[t]{4}{*}{ Moda do Aspecto 4} & D1 & 3 & 3 & 3 & 3 & 3 & 3 & 3 & 1 & 3 & 3 & 3 & 3 & 3 & 3 & 3 & 3 \\
\hline & D2 & $\star \star \star \star$ & $\star \star \star *$ & 1 & 1 & 1 & 1 & 1 & 1 & 1 & 1 & 1 & 1 & 1 & 1 & 1 & 1 \\
\hline & D4 & 1 & 1 & 1 & 1 & 1 & 1 & 1 & 1 & 1 & 1 & 1 & 1 & 1 & 1 & 1 & 1 \\
\hline & D8 & 3 & 3 & 3 & 3 & 3 & 3 & 3 & 3 & 3 & 3 & 3 & 3 & 3 & 3 & 3 & 3 \\
\hline
\end{tabular}


Quadro 4. Análise da variabilidade do padrão de movimento pela média dos IVPs de cada aspecto e pelo IVP de cada aspecto.

\begin{tabular}{|c|c|c|c|c|c|c|c|c|c|c|c|c|c|c|c|c|c|}
\hline \multirow{2}{*}{\multicolumn{2}{|c|}{ AVALIACCÃO DO PM }} & \multicolumn{16}{|c|}{ BLOCOS DE TENTATIVAS } \\
\hline & & 1 & 2 & 3 & 4 & 5 & 6 & 7 & 8 & 9 & 10 & 11 & 12 & 13 & 14 & TR1 & TR2 \\
\hline \multirow{4}{*}{$\begin{array}{l}\text { Média dos IVPs } \\
\text { de cada Aspecto }\end{array}$} & D1 & 0,3 & 0,0 & 0,2 & 0,0 & 0,1 & 0,1 & 0,1 & 0,1 & 0,1 & 0,1 & 0,0 & 0,0 & 0,1 & 0,1 & 0,0 & 0,1 \\
\hline & D2 & 0,2 & 0,1 & 0,1 & 0,1 & 0,1 & 0,1 & 0,0 & 0,1 & 0,1 & 0,1 & 0,0 & 0,0 & 0,1 & 0,1 & 0,0 & 0,0 \\
\hline & D4 & 0,1 & 0,1 & 0,1 & 0,1 & 0,1 & 0,1 & 0,1 & 0,1 & 0,1 & 0,1 & 0,1 & 0,1 & 0,0 & 0,0 & 0,1 & 0,1 \\
\hline & D8 & 0,1 & 0,1 & 0,0 & 0,1 & 0,0 & 0,1 & 0,1 & 0,0 & 0,0 & 0,0 & 0,0 & 0,1 & 0,0 & 0,0 & 0,1 & 0,0 \\
\hline \multirow[t]{4}{*}{ IVP do Aspecto 1} & D1 & 0,0 & 0,0 & 0,4 & 0,0 & 0,4 & 0,0 & 0,0 & 0,4 & 0,0 & 0,0 & 0,0 & 0,0 & 0,0 & 0,0 & $\mathrm{O}, \mathrm{O}$ & 0,0 \\
\hline & D2 & 0,0 & 0,0 & 0,4 & 0,0 & 0,4 & 0,0 & 0,0 & 0,0 & 0,0 & 0,0 & 0,0 & 0,0 & 0,0 & 0,0 & 0,0 & 0,0 \\
\hline & D4 & 0,0 & 0,0 & 0,4 & 0,0 & 0,0 & 0,0 & 0,0 & 0,0 & 0,0 & 0,0 & 0,0 & 0,0 & 0,0 & 0,0 & 0,0 & 0,0 \\
\hline & D8 & 0,0 & 0,0 & 0,0 & 0,0 & 0,0 & 0,0 & 0,0 & 0,0 & 0,0 & 0,0 & 0,0 & 0,0 & 0,0 & 0,0 & 0,0 & 0,0 \\
\hline \multirow[t]{4}{*}{ IVP do Aspecto 2} & D1 & 0,4 & 0,0 & 0,0 & 0,0 & 0,0 & 0,4 & 0,0 & 0,0 & 0,0 & 0,4 & 0,0 & 0,0 & 0,4 & 0,4 & 0,0 & 0,0 \\
\hline & D2 & 0,4 & 0,0 & 0,0 & 0,0 & 0,0 & 0,0 & 0,0 & 0,0 & 0,0 & 0,0 & 0,0 & 0,0 & 0,4 & 0,4 & 0,0 & 0,0 \\
\hline & D4 & 0,0 & 0,0 & 0,0 & 0,0 & 0,0 & 0,0 & 0,0 & 0,0 & 0,0 & 0,0 & 0,0 & 0,0 & 0,4 & 0,0 & 0,0 & 0,0 \\
\hline & D8 & 0,0 & 0,0 & 0,0 & 0,0 & 0,0 & 0,0 & 0,0 & 0,0 & 0,0 & 0,0 & 0,0 & 0,0 & 0,0 & 0,0 & 0,0 & 0,0 \\
\hline \multirow[t]{4}{*}{ IVP do Aspecto 3} & D1 & 0,0 & 0,0 & 0,0 & 0,0 & 0,0 & 0,0 & 0,0 & 0,0 & 0,0 & 0,0 & 0,0 & 0,0 & 0,0 & 0,0 & 0,0 & 0,4 \\
\hline & $\mathrm{D} 2$ & 0,0 & 0,0 & 0,0 & 0,0 & 0,0 & 0,0 & 0,0 & 0,0 & 0,0 & 0,0 & 0,0 & 0,0 & 0,0 & 0,0 & 0,0 & 0,0 \\
\hline & D4 & 0,0 & 0,0 & 0,0 & 0,0 & 0,0 & 0,0 & 0,0 & 0,0 & 0,0 & 0,0 & 0,0 & 0,0 & 0,0 & 0,0 & 0,0 & 0,0 \\
\hline & D8 & 0,4 & 0,4 & 0,0 & 0,0 & 0,0 & 0,4 & 0,4 & 0,0 & 0,0 & 0,0 & 0,0 & 0,0 & 0,0 & 0,0 & 0,4 & 0,0 \\
\hline \multirow[t]{4}{*}{ IVP do Aspecto 4} & & 0,0 & 0,0 & 0,0 & 0,0 & 0,0 & 0,0 & 0,0 & 0,0 & 0,0 & 0,0 & 0,0 & & 0,0 & 0,0 & 0 & 0,0 \\
\hline & $\mathrm{D} 2$ & 0,0 & 0,0 & 0,0 & 0,0 & 0,0 & 0,0 & 0,0 & 0,0 & 0,0 & 0,0 & 0,0 & 0,0 & 0,0 & 0,0 & 0,0 & 0,0 \\
\hline & & 0,0 & 0,0 & 0,0 & 0,0 & 0,0 & 0,0 & 0,0 & 0,0 & 0,0 & 0,0 & 0,0 & 0,0 & 0,0 & 0,0 & 0,0 & 0,0 \\
\hline & D8 & 0,0 & 0,0 & 0,0 & 0,0 & 0,0 & 0,0 & 0,0 & 0,0 & 0,0 & 0,0 & 0,0 & 0,0 & 0,0 & 0,0 & 0,0 & 0,0 \\
\hline
\end{tabular}

$\mathrm{Na}$ análise intragrupo do IVP por aspecto, o teste de Friedman identificou diferença no aspecto 1 para o D1 $[\mathrm{F}(\mathrm{n}=4, \mathrm{df}=14)=29,142, \mathrm{p}<0,01]$, para o D2 $[F(n=3, d f=14)=27,100, p<0,01]$ e para o D8 $[F(n=4, d f=14)=35,309, p<0,01]$. Por meio da observação do Quadro 4, é possível supor que, provavelmente, no $\mathrm{D} 1$, os blocos 3,5 e 8 tiveram variabilidade superior que os demais blocos, no D2, os blocos 3 e 5 tiveram variabilidade superior que os demais e o D8 apresentou diferenças intragrupos, porém não identificada no Quadro 4. O teste estatístico não detectou alteração nessa medida para o D4. No aspecto 2, o teste de Friedman identificou diferença para o $\mathrm{D} 1[\mathrm{~F}(\mathrm{n}=4, \mathrm{df}=14)=25,886, \mathrm{p}<0,02]$, o D2 $[\mathrm{F}(\mathrm{n}=3, \mathrm{df}=14)=28,000, \mathrm{p}<0,01]$, o D4 $[\mathrm{F}(\mathrm{n}=3, \mathrm{df}=14)=24,677, \mathrm{p}<0,03]$ e o $\mathrm{D} 8[\mathrm{~F}(\mathrm{n}=4$, $\mathrm{df}=14)=48,520, \mathrm{p}<0,01]$. Por meio da observação do Quadro 4, é possível supor que, provavelmente, no $\mathrm{D} 1$, os blocos 1, 6, 10, 13 e 14 tiveram variabilidade superior aos demais, no D2 os blocos 1, 13 e 14 tiveram variabilidade superior aos demais, no D4 o bloco 13 foi superior aos demais e no D8 houve diferença significativa intragrupo, porém não identificada no Quadro 4.

No aspecto 3, o teste de Friedman detectou diferença para o D2 $[\mathrm{F}(\mathrm{n}=3, \mathrm{df}=14)=27,605, \mathrm{p}<0,01]$, o $\mathrm{D} 4[\mathrm{~F}(\mathrm{n}=3, \mathrm{df}=14)=24,705, \mathrm{p}<0,03]$ e o $\mathrm{D} 8$
$[F(n=4, d f=14)=35,093, p<0,01]$. No D2 e no D4 houve diferença intragrupos, porém não identificada no Quadro 4. Por meio da observação do Quadro 4 é possível supor que, provavelmente, no D8, os blocos $1,2,6$ e 7 tiveram variabilidade superior aos demais. Não foi identificada, no D1, alteração significativa nessa medida.

No aspecto 4, o teste de Friedman detectou diferença para o D1 $[\mathrm{F}(\mathrm{n}=4, \mathrm{df}=14)=31,938, \mathrm{p}<0,01]$, o D2 $[\mathrm{F}(\mathrm{n}=3, \mathrm{df}=14)=28,825, \mathrm{p}<0,01]$, o D4 $[F(n=3, d f=14)=31,230, p<0,01]$ e o $D 8[F(n=4$, $\mathrm{df}=14)=42,976, \mathrm{p}<0,01]$. Em todos os grupos houve diferença, porém não identificada pela observação do Quadro 4.

\section{Testes de transferência e retenção}

$\mathrm{Na}$ análise intragrupo da média do escore, o teste de Friedman detectou diferença do último bloco de tentativas da aquisição para os testes no D1 $[\mathrm{F}(\mathrm{n}=4$, $\mathrm{df}=4)=10,683, \mathrm{p}<0,03]$ e no $\mathrm{D} 2[\mathrm{~F}(\mathrm{n}=3, \mathrm{df}=4)=$ $10,644, p<0,03]$. Em ambos os grupos, o procedimento de Bonferroni (teste de Wilcoxon) não foi capaz de identificar onde ocorreu a diferença devido ao ajuste do "p" resultante da utilização de medidas repetidas $(p>0,0125)$. Porém, por meio da observação da Figura 1, é possível supor que, provavelmente, a precisão do D1 e do D2 reduziu do último 
bloco da fase de aquisição para os dois blocos do TT. Não foi identificada diferença intergrupos, por meio do teste estatístico de Kruskal-Wallis na análise da média do escore $(\mathrm{p}>0,05)$.

$\mathrm{Na}$ análise do desvio-padrão do escore (Figura 2), o teste de Friedman não identificou diferença intragrupos do último bloco da aquisição para os testes, e o teste de Kruskal-Wallis não identificou diferença intergrupos ( $\mathrm{p}>0,05)$.

Com relação ao padrão de movimento, tanto ao analisar isoladamente a moda de cada aspecto quanto a somatória das modas de cada aspecto, o teste de Friedman não identificou diferenças intragrupos do último bloco da fase de aquisição para os testes, e o teste de Kruskal-Wallis não identificou diferença intergrupos para $\mathrm{p}<0,05$ (Quadro 3).

Outra análise conduzida através do teste de Friedman detectou diferença intragrupo para o D1 $[F(n=4, d f=2)=6,000, p<0,05]$ entre o último bloco da aquisição e o TR. Por meio do procedimento de Bonferroni (teste de Wilcoxon) não foi possível identificar entre quais blocos ocorreu a diferença ( $p>0,025)$, mas, por meio da observação do Quadro 4 , é possível supor que o último bloco da aquisição teve variabilidade superior ao bloco 1 do TR.

$\mathrm{Na}$ análise da média entre os IVPs de cada aspecto, o teste de Kruskal-Wallis identificou diferença intergrupos $\left[\mathrm{H}^{2}(7,829, \mathrm{df}=4) \mathrm{p}<0,05\right]$ no primeiro bloco do TR. Por meio de do procedimento de Bonferroni não foi possível identificar entre quais grupos ocorreu a diferença $(\mathrm{p}>0,0083)$. Porém, por meio da observação do Quadro 4, é possível supor que D4 e D8 tiveram superior variabilidade no padrão de movimento que D1 e D2.

$\mathrm{Na}$ análise dos IVPs por aspecto (Quadro 4), os testes de Friedman e de Kruskal-Wallis não detectaram diferenças intragrupo, entre o último bloco da aquisição e o TR, e intergrupo, respectivamente $(\mathrm{p}>0,05)$.

\section{DISCUSSÃO}

O objetivo deste estudo foi investigar o efeito de diferentes números de demonstrações na aprendizagem do arremesso de dardo de salão. Em linhas gerais, os resultados dos testes estatísticos, para as análises intra e intergrupos, tanto da média quanto do desvio-padrão do escore, não apresentaram alterações significativas ao longo da fase de aquisição, corroborando os achados de Feltz (10), o que é comum em estágios iniciais de aquisição de habilidades, nos quais os aprendizes buscam adquirir a idéia ou a forma do movimento $(10,12,19)$. Todos os grupos mantiveram o escore ao longo da aquisição, exceto o D1 que aumentou sua precisão, o que já é um indicativo de aprendizagem. Contudo, os principais resultados encontram-se no TR e no TT.

Ainda discutindo os resultados da análise do escore, tanto D1 quanto D2 reduziram a precisão do último bloco da aquisição para o bloco 1 do TT. Este resultado pode se relacionar aos números de demonstrações recebidos pelos dois grupos, ou seja, menos oportunidade para extrair detalhes importantes do movimento, que pode ter gerado um conjunto de informações limitado, o qual permitiu poucas alterações, e desfavorável a uma boa precisão em uma tarefa com características novas.

A análise dos resultados da avaliação do padrão de movimento na fase de aquisição e no TR confirmou a formação de uma representação cognitiva responsável pelo padrão de movimento (8). Desta maneira, é possível supor uma semelhança, intra e intergrupos, com relação à representação cognitiva que gera o movimento e que pode estar relacionada ao nível de complexidade da tarefa. Newell, Morris \& Scully (18), Carrol \& Bandura (8) e Laguna (14) sugerem que quanto maior o número de componentes de uma habilidade motora, maior a necessidade de demonstrações para que os aprendizes captem informações importantes e necessárias a um bom desempenho. Para a tarefa em questão se observou que uma demonstração foi suficiente, o que pode ser devido ao pequeno número de graus de liberdade da tarefa, sugerindo uma relação específica entre o número de demonstrações e a habilidade a ser aprendida (10). Contudo, essa é uma questão que ainda merece ser investigada em tarefas com níveis de maior complexidade.

A análise da moda de cada aspecto do padrão de movimento mostra que todos os grupos foram capazes de focalizar a atenção às características estruturais dos componentes do movimento e tiveram capacidade cognitiva suficiente para formular e reter a representação cognitiva da ação. Isto pode ser devido à interação entre a complexidade da tarefa e o nível de desenvolvimento motor dos voluntários do experimento (17). 
A redução da variabilidade do padrão de movimento, durante a fase de aquisição, do D1, do D2 e do D8, pode ter ocorrido pelo ganho de consistência no movimento (8) e continuou ocorrendo com D1 do último bloco da aquisição para o bloco 1 do TR. Este fato sustenta a predição de Bandura (2), que responsabiliza a prática por proporcionar momentos que promovem o reforço da representação cognitiva. Quanto à diferença intergrupo para a média do Índice de Variabilidade do Padrão de Movimento (IVP) entre os aspectos, no bloco 1 do TR, é possível sugerir que diferentes números de demonstrações, por meio de um modelo nas condições reais de práti$\mathrm{ca}$, podem permitir aos aprendizes captar diferentes informações (15). Isto pode ter formado representações cognitivas semelhantes em todos os grupos, porém com diferentes arranjos dessas informações, que podem ter promovido a capacidade dos sujeitos do D4 e o D8 de explorar variações no padrão de movimento. Os resultados das medidas do padrão de movimento vão ao encontro dos obtidos nas medidas de desempenho, porque tanto D4 quanto D8 não reduziram seu desempenho no TT. Sendo assim, é possível supor que o fornecimento de 4 ou 8 demonstrações na tarefa em questão permitiu aos aprendizes formarem uma representação cognitiva com maior flexibilidade em seu arranjo de informações e com a capacidade de experimentar novas alternativas no movimento, sem ter queda no desempenho. O efeito das características do modelo, sob condições reais ou adaptadas, merece mais investigações, especialmente na aprendizagem de tarefas com diferentes níveis de previsibilidade ambiental.

Por meio da análise dos IVPs por aspecto, os resultados mostraram que todos os grupos reduziram sua variabilidade durante a fase de aquisição, que se manteve no teste de retenção. Isto pode ter ocorrido pelo fortalecimento dos processos de detecção e correção dos erros, reforçando o plano de ação durante a prática.

Contudo, é possível dizer que as demonstrações no contexto real de ensino-aprendizagem da habilidade motora são importantes na formulação de uma representação cognitiva flexível, que permite com o aprendiz ajustar seus movimentos a situações com diferentes exigências.
Os resultados, extrapolados às situações reais, dão indícios de que para a aprendizagem de habilidades motoras, os professores/treinadores devem analisar as características dos alunos, da habilidade motora quanto à complexidade, do ambiente onde a habilidade será praticada e do modelo, para que o número de demonstrações seja mais efetivo.

\section{CORRESPONDÊNCIA}

\section{Herbert Ugrinowitsch}

Departamento de Esportes

Escola de Educação Física, Fisioterapia e Terapia

Ocupacional

Universidade Federal de Minas Gerais

Campus Pampulha

Avenida Presidente Carlos Luz, 4664

Pampulha, 31330-250

Belo Horizonte, Minas Gerais

Brasil

herbertu@eeffto.ufmg.br 


\section{REFERÊNCIAS}

1. Al-Abood S, Davids K, Bennet S J (2001). Specificity of task constraints and effects of visual demonstrations and verbal instructions in directing learner's search during skill acquisition. Journal of Motor Behavior 33: 295-305

2. Bandura A (1986). Social foundations of thought and action: a social cognitive theory. Englewood Cliffs: Prenctice-Hall

3. Barela AMF, Barela JA (1997). Restrições ambientais no arremesso de ombro. Motriz 3: 65-72

4. Bruzi AT, Palhares LR, Fialho JVAP, Benda RN, Ugrinowitsch H (2004). A influência do número de demonstrações na aprendizagem do arremesso de dardo de salão. In: Anais do XXVII Simpósio Internacional de Ciências do Esporte. São Paulo, 298

5. Carrol W, Bandura A (1982). The role of visual monitoring in observational learning of action patterns: making the unobservable observable. Journal of Motor Behavior 14: 153-167

6. Carrol W, Bandura A (1985). The role of timing of visual monitoring and motor rehearsal in observational learning of action patterns. Journal of Motor Behavior 17: 269-281

7. Carrol W, Bandura A (1987). Translating cognition into action: the role of visual guidance in observational learning. Journal of Motor Behavior 19: 385-398

8. Carrol W, Bandura A (1990). Representation guidance of action production in observational learning: a causal analysis. Journal of Motor Behavior 22: 85-97

9. Christina R (1989). Motor learning: futures lines of research. In Safrit MJ, Eckert HM (Ed.). The cutting edge in physical education and exercise science research. Champaign: Human Kinetics, 26-41

10. Feltz D (1982). The effect of age and number of demonstrations on modeling of form and performance. Research Quarterly for Exercise and Sport 53: 291-296

11. Fialho JVAP, Ugrinowitsch H (2004). O efeito da interferência contextual no treinamento de habilidades motoras esportivas. In Silami-Garcia E, Lemos K L M (eds.) Temas Atuais em Educação Física e Esportes IX. Belo Horizonte: Saúde, 21-35

12. Gentile A M (1972). A working model of skill acquisition with application to teaching. Quest 17: 3-23

13. Gimenez R, Ugrinowitsch H, Souza LL, Lima P, Magalhães D, Jaess AC (2002). Influência de um programa de atividade motora sobre padrões fundamentais de movimento em crianças de segunda infância. In Anais do II Congresso Latino-Americano da FIEP-UNIMEP. Piracicaba, 335-339

14. Laguna PL (1999). Effects of multiple correct model demonstration on cognitive representation development and performance accuracy in motor skill acquisition. Journal of Human Movement Studies 37: 55-86

15. Landers DM (1978). When and where to use demonstrations: suggestions for the practioners. Journal of Physical Education and Recreation 49: 65-67

16. Manoel EJ (1999). A dinâmica do estudo do comportamento motor. Revista Paulista de Educação Física 13: 52-61

17. McCullagh P, Weiss MR, Ross D (1989). Modeling considerations in motor skill acquisition and performance: an integrated approach. In Pandolf KB (ed.) Exercise and sport science reviews. Baltimore: Willians and Wilkins

18. Newell K, Morris L, Scully D (1985). Augmented information and the acquisition of skills in physical activity. In Terjung R (ed.) Exercise and Sports Sciences Review. New York: Macmillan, 235-261
19. Públio NS, Tani G, Manoel EJ (1995). Efeitos da demonstração e instrução verbal na aprendizagem de habilidades motoras da ginástica olímpica. Revista Paulista de Educação Física 9: 111-124

20. Tani G (1992). Contribuições da aprendizagem motora à Educação Física: uma análise crítica. Revista Paulista de Educação Física 2: 65-72

21. Tani G (1997). Esporte e técnica. In Tambucci PL, Mariz De Oliveira JG, Sobrinho JC (ed.) Esporte e Jornalismo. São Paulo: CEPEUSP, 33-38

22. Thomas JR, Nelson JK (2001). Métodos de pesquisa em atividade física. 3 edição, Porto Alegre: Artmed

23. Ugrinowitsch H (1999). Pesquisa de síntese em biodinâmica do movimento. In Anais do II Simpósio da Escola de Educação Física e Esportes da Universidade de São Paulo. São Paulo, 68

24. Ugrinowitsch H, Manoel EJ (1999). Interferência Contextual: variação de programa e parâmetro na aquisição da habilidade motora saque do voleibol. Revista Paulista de Educação Física 13: 197-216

25. Vincent WJ (1999). Statistics in kinesiology. 2 edition, Champaign: Human Kinetics

26. Weeks DL, Choi J (1992). Modeling the perceptual component of a coincident-timing skill: the influence of frequency of demonstration. Journal of Human Movement Studies 23: 201-213. 